\title{
Continuous version of the Choquet integral representation theorem
}

\author{
by \\ Piotr Puchata (Częstochowa)
}

\begin{abstract}
Let $E$ be a locally convex topological Hausdorff space, $K$ a nonempty compact convex subset of $E, \mu$ a regular Borel probability measure on $E$ and $\gamma>0$. We say that the measure $\mu \gamma$-represents a point $x \in K$ if $\sup _{\|f\| \leq 1}\left|f(x)-\int_{K} f d \mu\right|<\gamma$ for any $f \in E^{*}$. In this paper a continuous version of the Choquet theorem is proved, namely, if $P$ is a continuous multivalued mapping from a metric space $T$ into the space of nonempty, bounded convex subsets of a Banach space $X$, then there exists a weak* continuous family $\left(\mu_{t}\right)$ of regular Borel probability measures on $X \gamma$-representing points in $P(t)$. Two cases are considered: in the first case the values of $P$ are compact, while in the second they are closed. For this purpose it is shown (using geometrical tools) that the mapping $t \mapsto \operatorname{ext} P(t)$ is lower semicontinuous. Continuous versions of the Krein-Milman theorem are obtained as corollaries.
\end{abstract}

1. Introduction. The classical Minkowski-Carathéodory representation theorem states that each point of a compact convex set $K$ in $\mathbb{R}^{n}$ can be written as a convex combination of at most $n+1$ extreme points of $K$. This theorem was generalized by G. Choquet ([5]) who proved that each point $k$ of a compact, convex and metrizable subset $K$ of a locally convex Hausdorff topological space $X$ is a barycenter of a regular Borel probability measure $\mu_{k}$ on $X$, supported by the extreme points of $K$, i.e.

$$
f(k)=\int_{K} f d \mu_{k}
$$

for any $f \in X^{*}$, where $\mu_{k}(\operatorname{ext} K)=1$ and ext $K$ stands for the set of extreme points of $K$.

E. Bishop and K. de Leeuw ([3]) removed the metrizability assumption. G. A. Edgar ([7]) proved a noncompact version of the Choquet theorem. His result stated that the assertion of Choquet-Bishop-de Leeuw remained true for $K$ being a nonempty bounded, closed, convex and separable sub-

2000 Mathematics Subject Classification: 54C60, 54C65, 46A55, 46B22.

Key words and phrases: multivalued mapping, continuous selection, Choquet representation theorem, Radon-Nikodym property, extreme point, strongly exposed point. 
set of a Banach space $X$ having the Radon-Nikodym property (RNP for short). In [8] he improved this result by removing the separability condition. P. Mankiewicz [11] modified it by introducing a "separable extremal ordering", more natural and easier to use than the partial order defined by Edgar (see [4, p. 174]).

The purpose of this paper is to show that an analogue of the Choquet theorem holds for "moving" sets, which are values of a multivalued mapping from a metric space $T$ into suitable subsets of a Banach space $X$. We shall consider two cases.

In the first case the multivalued mapping $P: T \leadsto X$ is continuous with compact convex values in a separable Banach space $X$. In the second case $X$ is a separable, reflexive Banach space and $P: T \leadsto X$ is continuous with bounded closed convex values (recall that a reflexive Banach space has RNP). By the celebrated Michael theorem there exists a continuous selection $p: T \rightarrow X$ of $P$, that is, $p(t) \in P(t)$ for all $t \in T$. It will be shown that each $p(t)$ is then an "almost barycenter" of a regular Borel probability measure $\mu_{t}$ on $X$ such that $\mu_{t}(\operatorname{ext} P(t))=1$. In other words, for any continuous selection $p$ of the multifunction $P$ there exists a continuous (in the weak* topology) family $\left(\mu_{t}\right)_{t \in T}$ of measures "almost representing" the points $p(t)$. In both cases it is crucial that the multifunction $t \mapsto \operatorname{ext} P(t)$ is lower semicontinuous. As obvious corollaries we obtain continuous versions of the Krein-Milman theorem.

All the necessary information about multifunctions can be found in [9]; for the Choquet theorem see [1], [13] and [4] (noncompact case); Banach spaces with the Radon-Nikodym property are subject of the classics [4], [6]; properties of measures on metric spaces are investigated in [2] (where one can also find a chapter devoted to multivalued mappings).

2. Preliminaries. In this section we state several definitions and facts needed.

Definition 2.1. Let $X$ and $Y$ be topological spaces and $P: X \leadsto Y$ a set-valued map with nonempty values. We say that

(a) $P$ is lower semicontinuous (lsc) if the set

$$
P^{-}(U):=\{x \in X: P(x) \cap U \neq \emptyset\}
$$

is open whenever $U \subset Y$ is open;

(b) $P$ is upper semicontinuous (usc) if the set

$$
P^{-}(V):=\{x \in X: P(x) \subset U\}
$$

is closed whenever $V \subset Y$ is closed;

(c) $P$ is continuous if it is both lower and upper semicontinuous. 
Theorem 2.1 (Michael). Let $X$ be a paracompact space, $Y$ a Banach space, and $P: X \leadsto Y$ a lower semicontinuous multifunction with convex values. Then:

(a) for any $\varepsilon>0$ there exists a continuous function $p_{\varepsilon}: X \rightarrow Y$ such that $d\left(p_{\varepsilon}(x), P(x)\right)<\varepsilon$ for all $x \in X$; this function is called an $\varepsilon$-selection of $P$;

(b) if in addition the values of $P$ are closed, then there exists a continuous function $p: X \rightarrow Y$ such that $p(x) \in P(x)$; this function is called a continuous selection of $P$.

Now let $X$ be a locally convex topological Hausdorff space, and let $K$ be a compact convex subset of $X$. If $\mu$ is a regular Borel probability measure on $X$, we say that it is supported by the set $A \subset X$ (not necessarily closed) if $\mu(A)=1$.

Definition 2.2. Let $X, K$ and $\mu$ be as above, and let $\gamma>0$. We say that:

(a) $\mu$ represents a point $x \in K$ if

$$
f(x)=\int_{K} f d \mu \quad \text { for all } f \in X^{*} .
$$

This point, denoted by $r(\mu)$, is called the barycenter of $\mu$;

(b) $\mu \gamma$-represents $x \in K$ if

$$
\sup _{\|f\| \leq 1}\left|f(x)-\int_{K} f d \mu\right|<\gamma \quad \text { for all } f \in X^{*} .
$$

Theorem 2.2 (Choquet). Let $X, K$ and $\mu$ be as above and assume additionally that $K$ is metrizable. Then for any $x \in K$ there exists a regular Borel probability measure $\mu_{x}$ representing $x$ and such that $\mu_{x}(\operatorname{ext} K)=1$.

Recall that if the set ext $K$ is closed then the Choquet theorem is equivalent to the Krein-Milman theorem.

Theorem 2.3 (Edgar-Mankiewicz noncompact version of the Choquet theorem). Let $K$ be a (nonempty) closed bounded convex subset of a Banach space $X$ and suppose that $K$ has RNP. Then every point of $K$ is a barycenter of a regular Borel probability measure on $K$ supported by the set ext $K$.

3. Compact case. In this section we deal with multifunctions $P$ from $T$ into compact convex subsets of $X$.

We first establish the lower semicontinuity of the map whose values are the sets of extreme points of the compact convex sets which are values of $P$. Recall that an exposed point of a compact convex subset of a Banach space is a strongly exposed point of this subset. 
Proposition 3.1. Let $T$ be a metric space, $X$ a Banach space, and $P: T \leadsto X$ a continuous multifunction with compact convex values. Then the multifunction $t \mapsto \operatorname{ext} P(t)$ is lower semicontinuous.

Proof. Let $\left(t_{n}\right)$ be a sequence in $T$, convergent to a point $t_{0} \in T$. It is enough to show that for any $a_{0} \in \operatorname{ext} P\left(t_{0}\right)$ there exists a sequence $\left(a_{n}\right)$, $n \in \mathbb{N}$, such that $a_{n} \in \operatorname{ext} P\left(t_{n}\right)$ and $a_{n} \rightarrow a_{0}$ as $n \rightarrow \infty$.

Let $e_{0}$ be any exposed (in fact strongly exposed) point of $P\left(t_{0}\right)$. Then there exists a functional $f_{0} \in X^{*}$, with unit norm, strongly exposing $e_{0}$. The lower semicontinuity of $P$ yields a sequence $\left(x_{n}\right) \subset X$, convergent to $e_{0}$, with $x_{n} \in P\left(t_{n}\right)$. Fix a number $\gamma>0$ and define the slice

$$
R_{\gamma}\left(t_{n}\right):=\left\{x \in P\left(t_{n}\right): f_{0}(x)>c\left(f_{0}, P\left(t_{n}\right)\right)-\gamma\right\},
$$

where $c(\cdot, A)$ stands for the support function of the set $A$. Then there exists $n_{0} \in \mathbb{N}$ such that $R_{\gamma}\left(t_{n}\right) \cap \operatorname{ext} P\left(t_{n}\right) \neq \emptyset$ for every $n \geq n_{0}$. Indeed, suppose not. Then for each $n_{0} \in \mathbb{N}$ there exists $n \geq n_{0}$ for which ext $P\left(t_{n}\right) \subset$ $X \backslash R_{\gamma}\left(t_{n}\right)$. Hence there exists a subsequence $n_{k} \rightarrow \infty$ such that for each $e \in \operatorname{ext} P\left(t_{n_{k}}\right)$

$$
f_{0}(e)<c\left(f_{0}, P\left(t_{n_{k}}\right)\right)-\gamma
$$

By the Krein-Milman theorem the set $P\left(t_{n_{k}}\right)$ coincides with the closed convex hull of its extreme points, so in particular we have

$$
f_{0}\left(x_{n_{k}}\right) \leq c\left(f_{0}, P\left(t_{n_{k}}\right)\right)-\gamma, \quad k=1,2, \ldots
$$

Passing to the limit we obtain the inequality

$$
f_{0}\left(e_{0}\right) \leq c\left(f_{0}, P\left(t_{0}\right)\right)-\gamma,
$$

contradicting the fact that $e_{0}$ is strongly exposed.

Now let $\gamma=1 / m, m=1,2, \ldots$, and consider the slices $R_{1 / m}(\cdot)$. Then for each $m$ there exists $n_{m}$ such that for $n \geq n_{m}$ we have

$$
R_{1 / m}\left(t_{n}\right) \cap \operatorname{ext} P\left(t_{n}\right) \neq \emptyset \text {. }
$$

We can assume that $n_{m} \leq n<n_{m+1}$. For such $n$ choose $e_{n} \in R_{1 / m}\left(t_{n}\right) \cap$ ext $P\left(t_{n}\right)$, so that

$$
f_{0}\left(e_{n}\right) \geq c\left(f_{0}, P\left(t_{n}\right)\right)-1 / m \quad \text { for } n_{m} \leq n<n_{m+1} .
$$

By the upper semicontinuity of $P$ there exists a subsequence of $\left(e_{n}\right)$ (still denoted $\left(e_{n}\right)$ ) convergent to a point $\bar{e}_{0} \in P\left(t_{0}\right)$. As the values of $P$ are convex, we can use the relationship between the Hausdorff distance $h(A, B)$ of convex sets $A, B$ and their support functions:

$$
\sup _{\|f\| \leq 1}\left\{\left|c\left(f, P\left(t_{n}\right)\right)-c\left(f, P\left(t_{0}\right)\right)\right|\right\}=h\left(P\left(t_{n}\right), P\left(t_{0}\right)\right) .
$$

The compactness of $P(t), t \in T$, yields the continuity of $P$ in the Hausdorff metric, which gives us $h\left(P\left(t_{n}\right), P\left(t_{0}\right)\right) \rightarrow 0$ as $n \rightarrow \infty$, which in turn implies that $c\left(f, P\left(t_{n}\right)\right) \rightarrow c\left(f, P\left(t_{0}\right)\right)$ for any $f \in X^{*}$. But for $n_{m} \leq n \leq n_{m+1}$ we 
have $f_{0}\left(e_{n}\right) \geq c\left(f_{0}, P\left(t_{n}\right)\right)-1 / m$. Taking into account that $f_{0}\left(e_{n}\right)$ converges to $f_{0}(\bar{e})$ we finally get $e=\bar{e}_{0}$.

Now by the Lindenstrauss-Troyanski result ([10], [15], see also [4]) the set $P\left(t_{0}\right)$ equals the closed convex hull of its (strongly) exposed points, and by Milman's partial converse of the Krein-Milman theorem those points are dense in ext $P\left(t_{0}\right)$.

We are now ready to construct a desired sequence of extreme points. So let $a_{0}$ be any extreme point of $P\left(t_{0}\right)$. Fix $n_{1} \in \mathbb{N}$ and $e_{0}^{n_{1}} \in(\operatorname{st}) \exp P\left(t_{0}\right)$. There exists a sequence $\left(b_{n}^{1}\right)$ of extreme points of $P\left(t_{0}\right)$ convergent to $e_{0}^{n_{1}}$. Then there exists $n_{2}>n_{1}$ such that $\left\|e_{0}^{n_{1}}-b_{n}^{1}\right\|<1 / n_{1}$ for all $n \geq n_{2}$. Now take $e_{0}^{n_{2}} \in($ st $) \exp P\left(t_{0}\right)$ with $\left\|e_{0}^{n_{2}}-a_{0}\right\|<1 / n_{2}$ and a sequence $\left(b_{n}^{2}\right)$ of extreme points of $P\left(t_{n}\right)$ convergent to $e_{0}^{n_{2}}$. Then there exists $n_{3}>n_{2}$ such that $\left\|e_{0}^{n_{2}}-b_{n}^{2}\right\|<1 / n_{1}$ for all $n \geq n_{3}$. Repeating this procedure we obtain sequences $\left(b_{n}^{i}\right), b_{n}^{i} \in \operatorname{ext} P\left(t_{n}\right)$. Setting $a_{n}:=b_{n}^{i}$ we obtain the desired sequence.

REMARK. Tolstonogov and Finogenko proved (under the same assumptions) in [14] the lower semicontinuity of the map $t \mapsto \operatorname{clext} P(t)$, where "cl" stands for "closure". This result is equivalent to the above, but the method of proof presented here is of geometrical nature, in contrast to the topological methods they used. Incidentally, it seems that considering the map $t \mapsto \operatorname{ext} P(t)$ instead of $t \mapsto \operatorname{clext} P(t)$ is "in the spirit" of the Choquet theorem.

Now let $T$ be a metric space, and $X$ a separable Banach space. We denote by $\mathcal{M}(X)$ the regular Borel probability measures on $X$. We consider a continuous multifunction $P: T \leadsto X$ with (nonempty) compact convex values. The Michael selection theorem ensures the existence of a continuous selection $p$ of $P$. Fix such a $p$, and $\gamma>0$, and define the set-valued map $L: T \leadsto \mathcal{M}(X)$ by setting

$$
\begin{aligned}
& L(t):=\{\mu \in \mathcal{M}(X): \mu(\operatorname{ext} P(t))=1, \\
&\left.\sup _{\|f\| \leq 1}\left|f(p(t))-\int_{P(t)} f d \mu\right|<\gamma \text { for all } f \in X^{*}\right\} .
\end{aligned}
$$

The Choquet theorem guarantees that $L(t)$ is nonempty for all $t \in T$.

Proposition 3.2. The multifunction $L$ is lower semicontinuous.

Proof. It is enough to show that for any sequence $\left(t_{n}\right) \subset T$ convergent to $t_{0} \in T$ and for any nonempty, weak* closed subset $F$ of $\mathcal{M}(X)$, we have the implication $L\left(t_{n}\right) \subset F \Rightarrow L\left(t_{0}\right) \subset F$.

Take any element $\mu_{0}$ of $L\left(t_{0}\right)$. The set of discrete measures on clext $P\left(t_{0}\right)$ is dense in the set of measures supported by that set, so there exists a sequence $\left(m_{k}\right)$ of discrete measures (i.e. convex combinations of Dirac mea- 
sures), weak ${ }^{*}$ convergent to $\mu_{0}$. Hence there exists $k_{0}$ such that for all $k \geq k_{0}$ the measure $m_{k} \gamma$-represents the point $p\left(t_{0}\right)$. Each measure $m_{k}, k \geq k_{0}$, is a convex conbination

$$
m_{k}=\sum_{i=1}^{m} \lambda_{i} \delta_{a_{i}},
$$

where $a_{i} \in \operatorname{ext} P\left(t_{0}\right)$. As $\operatorname{ext} P(\cdot)$ is lower semicontinuous, for any $i=$ $1, \ldots, m$ there exists a sequence $\left(b_{n}^{i}\right), b_{n}^{i} \in \operatorname{ext} P\left(t_{n}\right)$, convergent to $a_{i}$. This means that for fixed $k$ the measures $\mu_{n}^{k}=\sum_{i=1}^{m} \lambda_{i} \delta_{b_{n}^{i}}$ converge to $m_{k}$ as $n \rightarrow \infty$. For $f \in X^{*}$ we also have

$$
\begin{aligned}
\mid f\left(p\left(t_{n}\right)\right)- & \int_{P\left(t_{n}\right)} f d \mu_{n}^{k} \mid \\
\leq & \left|f\left(p\left(t_{n}\right)\right)-f\left(p\left(t_{0}\right)\right)\right|+\left|f\left(p\left(t_{0}\right)\right)-\int_{P\left(t_{0}\right)} f d \mu_{0}\right| \\
& \quad+\left|\int_{P\left(t_{0}\right)} f d \mu_{0}-\int_{P\left(t_{0}\right)} f d m_{k}\right|+\left|\int_{P\left(t_{0}\right)} f d m_{k}-\int_{P\left(t_{n}\right)} f d \mu_{n}^{k}\right| .
\end{aligned}
$$

The first and the last terms on the right converge to zero. Since there exists $M>0$ such that $\sup \left\{\|y\|: y \in P\left(t_{0}\right)\right\} \leq M$, for $x \in P\left(t_{0}\right)$ we have $|f(x)| \leq M\left|f\left(M^{-1} x\right)\right| \leq M$. Taking into account that the sets supp $m_{k}$ and supp $\mu_{0}$ are subsets of $P\left(t_{0}\right)$ we obtain

$$
\begin{aligned}
\sup _{\|f\| \leq 1}\left|\int_{P\left(t_{0}\right)} f d m_{k}-\int_{P\left(t_{0}\right)} f d \mu_{0}\right| & =\sup _{\|f\| \leq 1}\left|\int_{P\left(t_{0}\right)} f d\left(m_{k}-\mu_{0}\right)\right| \\
& \leq M\left|\left(m_{k}-\mu_{0}\right)(1)\right| \underset{k \rightarrow \infty}{\longrightarrow} 0 .
\end{aligned}
$$

For fixed $k \geq k_{0}$ and for all $n \geq n_{0}$ we then have

$$
\left|f\left(p\left(t_{n}\right)\right)-\int_{P\left(t_{n}\right)} f d \mu_{n}^{k}\right|<\gamma .
$$

By construction, the measure $\mu_{n}^{k}$ is supported by the set of extreme points of $P\left(t_{n}\right)$, so for $n \geq n_{0}$ it belongs to $L\left(t_{n}\right)$ and thus to $F$. Passing to the limit gives $\mu_{0} \in F$, proving the lower semicontinuity of the multifunction $L$.

Corollary 3.1. The multifunction

$$
\begin{aligned}
& \operatorname{cl} L(t)=\{\mu \in \mathcal{M}(X): \mu(\operatorname{cl} \operatorname{ext} P(t))=1, \\
&\left.\sup _{\|f\| \leq 1}\left|f(p(t))-\int_{P(t)} f d \mu\right|<\gamma \text { for all } f \in X^{*}\right\}
\end{aligned}
$$

is lower semicontinuous.

COROLlary 3.2. There exists a continuous selection $\bar{l}: T \rightarrow \mathcal{M}(X)$ of the multifunction $\mathrm{cl} L$. 
The set of extreme points of a compact convex set need not be closed, so in general the values of the multifunction $L$ are not closed. In particular we cannot expect $L$ to have continuous selections. However, there exist approximate continuous selections, as stated in the next result.

We denote by $\mathcal{H}$ the Hilbert cube, and $\mathcal{M}(\mathcal{H})$ stands for the regular Borel probability measures on $\mathcal{H}$; this is a compact separable metric space.

Theorem 3.1 (continuous version of the Choquet theorem). Let $T$ be a metric space, $X$ a separable Banach space, and $P: T \leadsto X$ a continuous multifunction with compact convex values. Let $p$ be a continuous selection of $P$, and $\gamma>0$. Define $L: T \leadsto \mathcal{M}(X)$ by

$$
\begin{aligned}
L(t):=\{\mu \in \mathcal{M}(X): & \mu(\operatorname{ext} P(t))=1, \\
& \left.\sup _{\|f\| \leq 1}\left|f(p(t))-\int_{P(t)} f d \mu\right|<\gamma \text { for all } f \in X^{*}\right\} .
\end{aligned}
$$

Then for any $\delta>0, L$ admits a continuous $\delta$-selection.

Proof. We adopt Michael's classical method. There exists a continuous mapping $\widehat{\phi}: \mathcal{M}(X) \rightarrow \mathcal{M}(\mathcal{H})$ (see [2, pp. 483-485]). The Polish space $\mathcal{M}(X)$ is metrizable by

$$
d\left(\mu_{1}, \mu_{2}\right)=\varrho_{\mathcal{H}}\left(\widehat{\phi}\left(\mu_{1}\right), \widehat{\phi}\left(\mu_{2}\right)\right)=\sum_{j=1}^{\infty} \frac{1}{2^{j}}\left|\left(\widehat{\phi}\left(\mu_{1}\right)-\widehat{\phi}\left(\mu_{2}\right)\right)\left(\zeta_{j}\right)\right|,
$$

where $\zeta_{j}, j=1,2, \ldots$, are from a dense set in $C(\mathcal{H})$, the space of continuous functions on the Hilbert cube. We start by fixing: a dense set $\left(\zeta_{n}\right)_{n=1}^{\infty}$ in $C(\mathcal{H})$, numbers $\delta>0$ and $N \in \mathbb{N}$ with $\sum_{j=N+1}^{\infty} 1 / 2^{j}<\delta / 4$, a point $t_{0} \in T$ and a measure $\mu_{0} \in L\left(t_{0}\right)$. The mapping $\mu \mapsto\left|\left(\widehat{\phi}(\mu)-\widehat{\phi}\left(\mu_{0}\right)\right)\left(\zeta_{j}\right)\right|$ is continuous for (any) $\mu_{0}$ and any fixed $j \in \mathbb{N}$, so the set

$$
V\left(\mu_{0}, \zeta_{1}, \ldots, \zeta_{N}, \delta\right):=\left\{\mu: \sum_{j=1}^{N} \frac{1}{2^{j}}\left|\left(\widehat{\phi}(\mu)-\widehat{\phi}\left(\mu_{0}\right)\right)\left(\zeta_{j}\right)\right|<\frac{\delta}{2}\right\}
$$

is open. The lower semicontinuity of $L$ implies that

$$
U\left(t_{0}, \mu_{0}\right):=L^{-1}(V)=\{t \in T: L(t) \cap V \neq \emptyset\}
$$

is an open neighborhood of $t_{0}$. Using the lower semicontinuity of $L$ again we obtain an open cover $\left\{U\left(t_{\alpha}, \mu_{\alpha}\right)\right\}_{\alpha \in I}$ of $T$. Denote by $e_{\alpha}(\cdot)$ a locally finite partition of unity subordinate to this covering. Our candidate for a continuous $\delta$-selection $l_{\delta}$ of $L$ is

$$
l_{\delta}(t):=\sum_{\alpha \in I} e_{\alpha}(t) \mu_{\alpha}
$$

Fix $t \in T$ and set $\left\{\alpha: e_{\alpha}(t)>0\right\}:=\left\{\alpha_{1}, \ldots, \alpha_{k}\right\}$; then $t \in \operatorname{supp} e_{\alpha_{i}} \subset$ $U\left(t_{\alpha_{i}}, \mu_{\alpha_{i}}\right)$, so the intersection of $L(t)$ with the ball of radius $\delta$, centred at 
$\mu_{\alpha_{i}}$, is nonempty. Let $\bar{\mu}_{i}$ belong to this intersection, so $d\left(\bar{\mu}_{i}, \mu_{\alpha_{i}}\right)<\delta$. The point $\bar{l}_{\delta}(t):=\sum_{i=1}^{k} e_{\alpha_{i}}(t) \bar{\mu}_{i}$ lies in the convex set $L(t) \cap V$. The assertion of the theorem follows from the following sequence of inequalities:

$$
\begin{aligned}
d\left(l_{\delta}(t), L(t)\right) & \leq d\left(l_{\delta}(t), \bar{l}_{\delta}(t)\right)=\varrho_{\mathcal{H}}\left(\widehat{\phi}\left(l_{\delta}(t)\right), \widehat{\phi}\left(\bar{l}_{\delta}(t)\right)\right) \\
& \leq \sum_{j=1}^{\infty} \frac{1}{2^{j}}\left|\left(\sum_{\alpha \in I} e_{\alpha}(t) \widehat{\phi}\left(\mu_{\alpha}\right)-\sum_{i=1}^{k} e_{\alpha_{i}}(t) \widehat{\phi}\left(\bar{\mu}_{i}\right)\right)\left(\zeta_{j}\right)\right| \\
& \leq \sum_{j=1}^{\infty} \frac{1}{2^{j}} \sum_{i=1}^{k} e_{\alpha_{i}}(t)\left|\left(\widehat{\phi}\left(\mu_{\alpha_{i}}\right)-\widehat{\phi}\left(\bar{\mu}_{i}\right)\right)\left(\zeta_{j}\right)\right| \\
& \leq \sum_{j=1}^{N} \frac{1}{2^{j}} \sum_{i=1}^{k}\left|\left(\widehat{\phi}\left(\mu_{\alpha_{i}}\right)-\widehat{\phi}\left(\bar{\mu}_{i}\right)\right)\left(\zeta_{j}\right)\right|+\sum_{j=N+1}^{\infty} \frac{1}{2^{j}} \\
& <\sum_{i=1}^{k} e_{\alpha_{i}}(t)\left(\sum_{j=1}^{N}\left|\left(\widehat{\phi}\left(\mu_{\alpha_{i}}\right)-\widehat{\phi}\left(\bar{\mu}_{i}\right)\right)\left(\zeta_{j}\right)\right|\right)+\frac{\delta}{4} \\
& <\delta / 2+\delta / 4<\delta .
\end{aligned}
$$

Corollary 3.3 (continuous version of the Krein-Milman theorem). Let $X, P$ and $p$ be as above. Then for any $\gamma>0$ there exists a continuous family $\left(\mu_{t}\right)_{t \in T}$ of measures on $X$ such that $\mu_{t}$ is supported by the closure of the set of extreme points of $P(t)$ and $\gamma$-represents the point $p(t)$.

4. Noncompact case. In this section we consider the case when the values of the multifunction are noncompact. We then have to impose more assumptions on both the Banach space $X$ and the multifunction $P$.

Recall that $x \in K$ is a denting point if for each $\varepsilon>0 x \notin \operatorname{clcv}\left(K \backslash U_{\varepsilon}(x)\right)$, where $U_{\varepsilon}(x)$ denotes the $\varepsilon$-neighbourhood of $x$.

Theorem 4.1. Let $T$ be a metric space, $X$ a reflexive Banach space, and $P: T \leadsto X$ a multifunction satisfying the following conditions:

(a) $P$ is continuous;

(b) for each $t \in T$ the set $P(t)$ is bounded, closed and convex;

(c) each extreme point of $P(t)$ is its denting point.

Then the multifunction $t \mapsto \operatorname{ext} P(t)$ is lower semicontinuous.

Proof. Exactly as in the compact case we construct the slice $R_{\gamma}(\cdot)$ and show that it is nonempty, replacing "Krein-Milman theorem" with "KreinMilman property", obtaining in the same way a sequence $\left(e_{n}\right)$ of extreme points belonging for $n_{n} \leq n \leq n_{m+1}$ both to the set ext $P\left(t_{n}\right)$ and the slice $R_{1 / m}\left(t_{n}\right)$. By the upper semicontinuity of $P$ this sequence is bounded and has a subsequence (still denoted by $\left(e_{n}\right)$ ) convergent to a point $\bar{e}_{0} \in P\left(t_{0}\right)$. 
The upper semicontinuity of $P$ implies its Hausdorff upper semicontinuity, so we can write

$$
\sup \left\{c\left(f, P\left(t_{n}\right)\right)-c\left(f, P\left(t_{0}\right)\right):\|f\| \leq 1\right\}=h^{*}\left(P\left(t_{n}\right), P\left(t_{0}\right)\right) \underset{n \rightarrow \infty}{\longrightarrow} 0,
$$

where $h^{*}(A, B)=\sup \{d(a, B): a \in A\}$. Thus $c\left(f, P\left(t_{n}\right)\right) \rightarrow c\left(f, P\left(t_{0}\right)\right)$ as $n \rightarrow \infty$ and (as in the compact case) we can conclude that $\bar{e}_{0}=e_{0}$.

We have thus constructed the sequence $\left(e_{n}\right), e_{n} \in \operatorname{ext} P\left(t_{n}\right)$, weakly convergent to the point $e_{0} \in \operatorname{stexp} P\left(t_{0}\right)$. Moreover, we have

$$
d\left(e_{n}, P\left(t_{0}\right)\right) \leq h^{*}\left(P\left(t_{n}\right), P\left(t_{0}\right)\right) \underset{n \rightarrow \infty}{\longrightarrow} 0,
$$

so there exists a sequence $\left(b_{n}\right) \subset P\left(t_{0}\right)$ with $\left\|b_{n}-e_{n}\right\| \rightarrow 0$. This yields weak convergence of $\left(b_{n}\right)$ to $e_{0}$, which in turn implies (as $e_{0}$ is strongly exposed) that $\left(b_{n}\right)$ converges to $e_{0}$ in norm. Thus $\left\|e_{n}-e_{0}\right\| \rightarrow 0$ as $n \rightarrow \infty$.

It turns out that the set of strongly exposed points of $P(t), t \in T$, is dense in ext $P(t)$. Indeed, suppose that this is not the case and consider a slice of $P(t)$ with norm diameter $\varepsilon$ containing some $e \in \operatorname{ext} P(t)$. None of the strongly exposed points of $P(t)$ belongs to the slice, so we have $P(t)=$ clcv stexp $P(t)$. This contradicts the fact that $e$ is a denting point.

The rest of the proof proceeds as in the compact case.

Now we are able to formulate a continuous version of the Choquet theorem and its corollary.

THEOREM 4.2 (continuous version of the noncompact Choquet theorem). Let $T$ be a metric space, $X$ a separable reflexive Banach space, and $P: T \leadsto X$ a multifunction satisfying the following conditions:

(a) $P$ is continuous;

(b) for each $t \in T$ the set $P(t)$ is bounded, closed and convex;

(c) each extreme point of $P(t)$ is its denting point.

Let $p$ be a continuous selection of $P$, let $\gamma>0$, and define $L: T \leadsto \mathcal{M}(X)$ by

$$
\begin{aligned}
& L(t):=\{\mu \in \mathcal{M}(X): \mu(\operatorname{ext} P(t))=1, \\
&\left.\sup _{\|f\| \leq 1}\left|f(p(t))-\int_{P(t)} f d \mu\right|<\gamma \text { for all } f \in X^{*}\right\} .
\end{aligned}
$$

Then for any $\delta>0$ there exists a continuous function $l_{\delta}: T \rightarrow \mathcal{M}(X)$ which is a $\delta$-selection of $L$.

COROLlary 4.1 (continuous version of the noncompact Krein-Milman theorem). Let T, $X, P$ and $p$ be as above. Then for any $\gamma>0$ there exists a continuous family $\left(\mu_{t}\right)_{t \in T}$ of measures on $X$ such that $\mu_{t}$ is supported by the closure of the set of extreme points of $P(t)$ and $\gamma$-represents the point $p(t)$. 
The proofs of these results are identical to the ones given in the previous section.

Acknowledgments. The author would like to thank Andrzej Fryszkowski, Piotr Mankiewicz and Kazimierz Brągiel for discussions.

\section{References}

[1] E. M. Alfsen, Compact Convex Sets and Boundary Integrals, Springer, 1971.

[2] Ch. D. Aliprantis and K. C. Border, Infinite Dimensional Analysis. A Hitchhiker's Guide, 2nd ed., Springer, 1999.

[3] E. Bishop and K. de Leeuw, The representations of linear functionals by measures on sets of extreme points, Ann. Inst. Fourier (Grenoble) 9 (1959), 305-331.

[4] R. D. Bourgin, Geometric Aspects of Convex Sets with the Radon-Nikodym Property, Lecture Notes in Math. 993, Springer, 1983.

[5] G. Choquet, Existence et unicité des représentations intégrales au moyen des points extrémaux dans les cônes convexes, Sém. Bourbaki (Dec. 1956), exp. 139, 15 pp.

[6] J. Diestel and J. J. Uhl, Jr., Vector Measures, Amer. Math. Soc., Providence, RI, 1977.

[7] G. A. Edgar, A noncompact Choquet theorem, Proc. Amer. Math. Soc. 49 (1975), 354-358.

[8] - Extremal integral representations, J. Funct. Anal. 23 (1976), 145-161.

[9] Sh. Hu and N. S. Papageorgiou, Handbook of Multivalued Analysis. Vol. I: Theory, Kluwer, 1998.

[10] J. Lindenstrauss, On operators which attain their norm, Israel J. Math. 3 (1963), 139-148.

[11] P. Mankiewicz, A remark on Edgar's extremal integral representation theorem, Studia Math. 63 (1978), 259-265.

[12] E. Michael, Continuous selections I, Ann. of Math. 63 (1956), 361-382.

[13] R. R. Phelps, Lectures on Choquet's theorem, 1st ed., Van Nostrand Math. Stud. 7, 1966, 2nd ed., Lecture Notes in Math. 1757, Springer, 2000.

[14] A. A. Tolstonogov and I. A. Finogenko, On functional-differential inclusions in a Banach space with non-convex right-hand side, Dokl. Akad. Nauk. SSSR 254 (1980), 45-49 (in Russian).

[15] S. L. Troyanski, On locally uniformly convex and differentiable norms in certain non-separable Banach spaces, Studia Math. 37 (1971), 173-180.

Institute of Mathematics and Computer Science

Technical University of Częstochowa

Dąbrowskiego 73

42-200 Częstochowa, Poland

E-mail: ppuchala@imi.pcz.pl

Received March 22, 2004

Revised version December 9, 2004 